$\begin{array}{cc}\text { ACADEMIA ROMÂNĂ } & \text { Rev. Roum. Chim., } \\ \text { 2020, 65(3), 239-246 }\end{array}$

\title{
GATIFLOXACIN-1,2,3-TRIAZOLE-ISATIN HYBRIDS TETHERED THROUGH METHYLENE AND ACETYL AND THEIR ANTIBACTERIAL ACTIVITIES
}

\author{
Hua GUO* and Quan-Ping DIAO \\ School of Chemistry and Life Science, Anshan Normal University
}

Received October 21, 2019

In this work, a series of gatifloxacin-1,2,3-triazole-isatin hybrids tethered with methylene and acetyl (8a-n) were designed and synthesized, and the antibacterial activity profiles together with cytotoxicity were also investigated. The preliminary results indicated that all of the hybrids showed promising activity against a panel of Gram-positive and Gram-negative bacteria with MIC values in a range of $\leq 0.03$ to $64 \mu \mathrm{g} / \mathrm{mL}$. The cytotoxicity results demonstrated all hybrids displayed acceptable cytotoxicity towards VERO cells. Among these 14 hybrids, 8b and $8 \mathrm{~g}$ with low cytotoxicity were no inferior to the parent gatifloxacin against Gram-positive and Gram-negative pathogens. Furthermore,<smiles>[R7]c1ccc2c(c1)N(Cc1cn(CC(=O)N3CCN(c4cc5c(cc4F)c(=O)c(C(=O)O)cn5C4CC4)CC3)nn1)C(=O)C2=[R]</smiles>
we discussed the structure-activity relationship and structure-cytotoxicity relationship so as to point out the direction for further rationale design and modification of this series of hybrids.

\section{INTRODUCTION}

Gram-positive and Gram-negative pathogens usually bring out the infections, even cause the death if there is no effective treatment. ${ }^{1,2}$ In the last several decades, antibiotics such as fluoroquinolones are used commonly to battle with bacterial infections, but the emergency and widely spread of drug-resistant bacteria make pathogens less and less susceptible to the antibiotics used currently. ${ }^{3,4}$ Drug-resistant bacteria have already been a knotty problem in the world healthcare system as evidenced by that roughly 700,000 drug-resistant pathogens related deaths occur around the world annually, and the deaths may increase to 10 million in the middle of this century if drug-resistant bacteria could not get effective control. ${ }^{5,6}$ Therefore, it is urgent to develop antibacterial agents which are more effective and sensitive to both drug-susceptible and drug-resistant organisms.
Gatifloxacin, a fourth generation of fluoroquinolone, which could inhibit the bacterial enzymes DNA gyrase and topoisomerase IV, possesses excellent broad-spectrum antibacterial activity against both Gram-positive and Gram-negative pathogens. However, gatifloxacin has not been used for the treatment of bacterial infections due to the significant side effects including dysglycemia. ${ }^{7,8}$ In recent years, various gatifloxacin derivatives were prepared and the antimicrobial activities were tested in order to obtain the compounds with the illustrious antibacterial activity and the reduced toxicity. ${ }^{7-9}$ Among them, the alkyl tethered gatifloxacin-1,2,3triazole-isatin hybrids $\mathbf{1}$ showed promising antibacterial activity and low cytotoxicity. Further study revealed that the linker between fluoroquinolone and 1,2,3-triazole moieties, as well as the carbon spacer between 1,2,3-triazole and isatin moiety influenced the activity and cytotoxicity significantly. The linker ethylene was more favorable than propylene between

\footnotetext{
*Corresponding author: m13704127672@163.com
} 
fluoroquinolone and 1,2,3-triazole motifs, suggesting the shorter linker was preferred. ${ }^{10-18}$ Moreover, $\mathrm{N}$-acylated fluoroquinolone derivatives showed enhanced antibacterial activity and reduced cytotoxicity when compared with the parent, indicating acetyl moiety might be an excellent linker. ${ }^{19-21}$

In this study, methylene group was inserted as a linker between isatin and 1,2,3-triazole motifs, and acetyl moiety was introduced a linker between gatifloxacin and 1,2,3-triazole moieties, respectively. All the synthesized gatifloxacin-1,2,3triazole-isatin hybrids tethered with methylene and acetyl were screened for their in vitro antibacterial activity against both drug-sensitive and drugresistant pathogens, as well as the cytotoxicity towards VERO cells. Our primary objective was to identify the optimal linkers between isatin and 1,2,3-triazole motifs as well as between gatifloxacin and 1,2,3-triazole moieties so as to facilitate the development of the drug candidates with higher efficiency and lower toxicity. The illustration of the design strategy is depicted in Figure 1.

\section{RESULTS AND DISCUSSION}

The synthetic route for methylene and acetyl tethered gatifloxacin-1,2,3-triazole-isatin hybrids 8a-n was described in Scheme 1. Isatin/5- fluoroisatin/5-methylisatin/7-fluoroisatin/5,7-dichloroisatin 1a-e were alkylated with propargyl bromide in presence of potassium carbonate $\left(\mathrm{K}_{2} \mathrm{CO}_{3}\right)$, providing $N$-propargyl isatin intermediates 2a-e. ${ }^{22,23}$ Then, intermediates 3a-i were obtained by the condensation of $N$-propargyl isatins 2a-e with the alkoxyamine hydrochlorides. Later, 2-bromoacetic acid $\mathbf{4}$ was treated with sodium azide, giving 2-azidoacetic acid 5, which was then reacted with dicyclohexylcarbodiimide (DCC) and $N$-hydroxysuccinimide (NHS) to get succinimidyl ester $\mathbf{6}^{24}$ Condensation of gatifloxacin with succinimidyl ester $\mathbf{6}$ in presence of $\mathrm{N}, \mathrm{N}$-diisopropylethylamine (DIPEA) yielded 2-azidoacetyl gatifloxacin 7. Finally, cyclization of $N$-propargyl isatin intermediates $2 \mathbf{a}-\mathbf{e}$ or 3a-i with 2-azidoacetyl gatifloxacin 7 in presence of copper acetate $\left(\mathrm{Cu}(\mathrm{OAc})_{2}\right)$ provided the desired acetyl tethered gatifloxacin-1,2,3-triazole-isatin hybrids 8a-n. ${ }^{18}$

The chemical structures and yields of acetyl tethered gatifloxacin-1,2,3-triazole-isatin hybrids 8a-n were listed in Table 1. From Table 1, it can be seen that the yields of the hybrids except $\mathbf{8 e}$ (yield: $22 \%$ ) were in a range of $43 \%$ to $79 \%$, and the low yield for $\mathbf{8 e}$ might be attributed to the electron deficiency (two chlorine atoms) which reduced the reactivity of propargyl at N-1 position of isatin moiety.

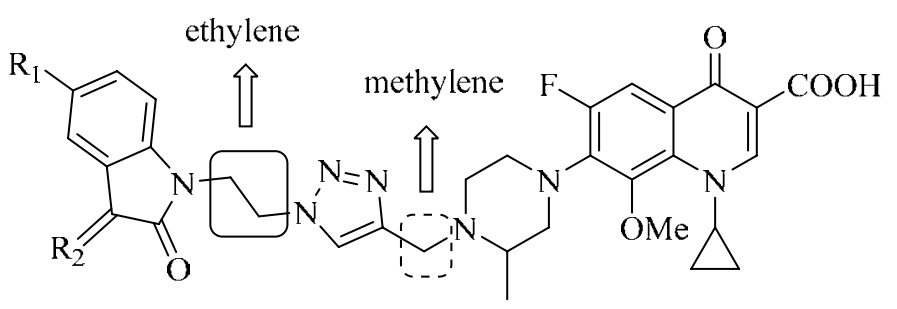

hybrids 1

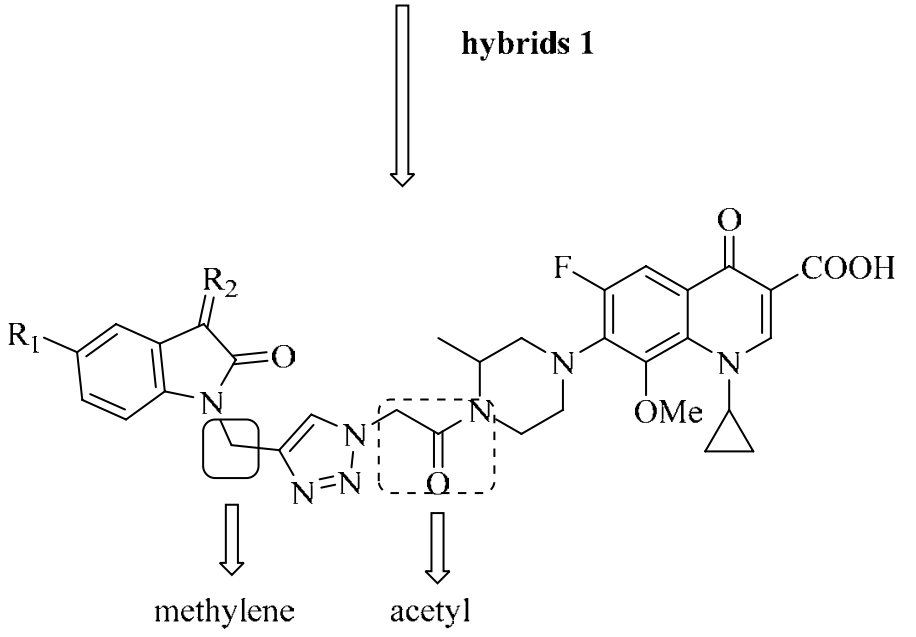

Fig. 1 - The design strategy of methylene and acetyl tethered gatifloxacin-1,2,3-triazole-isatin hybrids. 


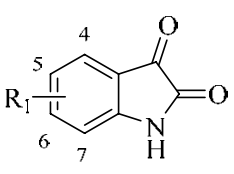

1

$$
\begin{aligned}
& \text { 1a: } \mathrm{R}_{1}=\mathrm{H} ; \\
& \text { 1b: } \mathrm{R}_{\mathrm{l}}=5-\mathrm{F} ; \\
& \text { 1c: } \mathrm{R}_{\mathrm{l}}=5-\mathrm{Me} \\
& \text { 1d: } \mathrm{R}_{\mathrm{l}}=7-\mathrm{F} ; \\
& \text { 1e: } \mathrm{R}_{\mathrm{l}}=5,7-\mathrm{diCl}
\end{aligned}
$$<smiles>[R]c1ccc2c(c1)N(CC#C)C(=O)C2=O</smiles>

2<smiles></smiles>

3 2a: $\mathrm{R}_{\mathrm{l}}=\mathrm{H}$;

2b: $\mathrm{R}_{\mathrm{l}}=5-\mathrm{Me}$;

2c: $\mathrm{R}_{\mathrm{l}}=5-\mathrm{F}$;

2d: $\mathrm{R}_{1}=7-\mathrm{F}$

2e: $\mathrm{R}_{\mathrm{l}}=5,7-\mathrm{diCl}$ 3a $\mathrm{R}_{1}=\mathrm{H}, \mathrm{R}_{2}=\mathrm{NOMe}$

3b: $\mathrm{R}_{1}=5-\mathrm{F}, \mathrm{R}_{2}=\mathrm{NOMe}$;

3c: $\mathrm{R}_{1}=7-\mathrm{F}, \mathrm{R}_{2}=\mathrm{NOMe}$

3d: $\mathrm{R}_{1}=\mathrm{H}, \mathrm{R}_{2}=\mathrm{NOH}$;

3e: $\mathrm{R}_{1}=5-\mathrm{F}, \mathrm{R}_{2}=\mathrm{NOH}$;

3f: $\mathrm{R}_{1}=5-\mathrm{Me} \mathrm{R}_{2}=\mathrm{NOH}$;

3g: $\mathrm{R}_{1}=\mathrm{H}, \mathrm{R}_{2}=\mathrm{NOEt}$

3h: $\mathrm{R}_{1}=5-\mathrm{F}, \mathrm{R}_{2}=$ NOEt;

3i: $\mathrm{R}_{1}=5-\mathrm{Me} \mathrm{R}_{2}=\mathrm{NOEt}$.<smiles>COc1c(N2CCN(C(=O)CN)C(C)C2)c(F)cc2c(=O)c(C(=O)O)cn(C3CC3)c12</smiles>

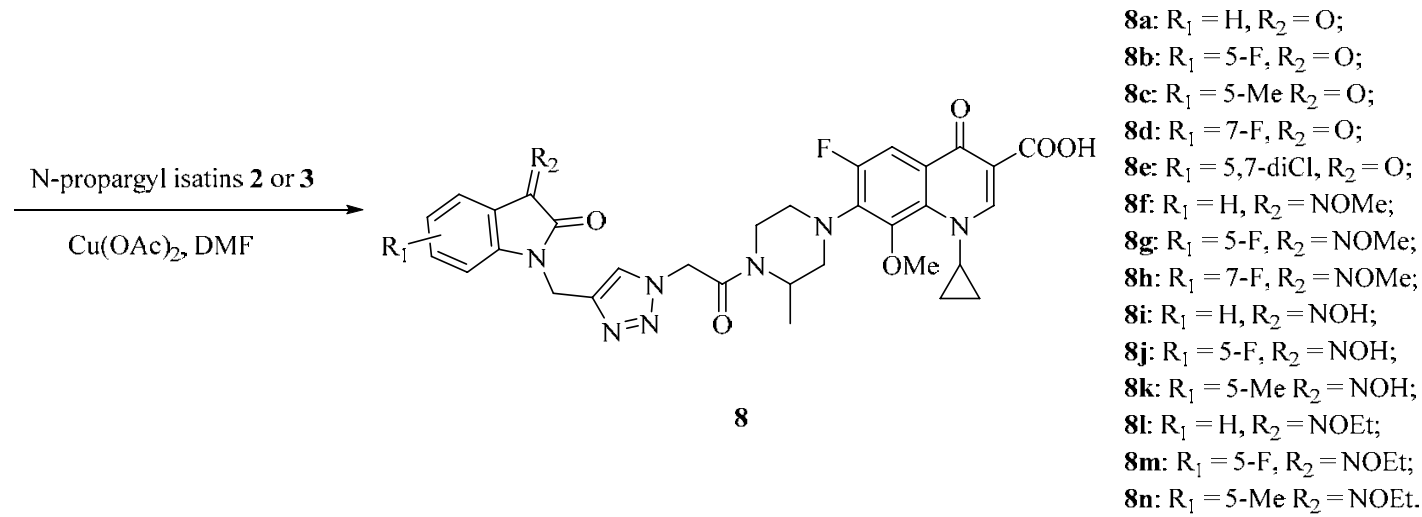

Scheme 1 - Synthesis of methylene and acetyl tethered gatifloxacin-1,2,3-triazole-isatin hybrids 8a-n.

Table 1

Chemical structures and yields of gatifloxacin-1,2,3-triazole-isatin hybrids 8a-n<smiles>[R]c1ccc2c(c1)C([R])C(=O)N2Cc1cn(CC(=O)N2CCN(c3cc4c(cc3F)c(=O)c(C(=O)O)cn4C3CC3)CC2)nn1</smiles>

8

\begin{tabular}{cccc}
\hline Compd. & $\mathbf{R}_{\mathbf{1}}$ & $\mathbf{R}_{\mathbf{2}}$ & Yield (\%) \\
\hline $\mathbf{8 a}$ & $\mathrm{H}$ & $\mathrm{O}$ & $63 \%$ \\
$\mathbf{8 b}$ & $5-\mathrm{F}$ & $\mathrm{O}$ & $71 \%$ \\
$\mathbf{8 c}$ & $5-\mathrm{Me}$ & $\mathrm{O}$ & $59 \%$ \\
$\mathbf{8 d}$ & $7-\mathrm{F}$ & $\mathrm{O}$ & $43 \%$ \\
$\mathbf{8 e}$ & $5,7-\mathrm{diCl}$ & $\mathrm{O}$ & $22 \%$ \\
$\mathbf{8 f}$ & $\mathrm{H}$ & $\mathrm{NOMe}$ & $79 \%$ \\
$\mathbf{8 g}$ & $5-\mathrm{F}$ & $\mathrm{NOMe}$ & $68 \%$ \\
\hline
\end{tabular}


Table 1 (continued)

\begin{tabular}{cccc}
\hline $\mathbf{8 h}$ & $7-\mathrm{F}$ & $\mathrm{NOMe}$ & $57 \%$ \\
$\mathbf{8 i}$ & $\mathrm{H}$ & $\mathrm{NOH}$ & $47 \%$ \\
$\mathbf{8 j}$ & $5-\mathrm{F}$ & $\mathrm{NOH}$ & $56 \%$ \\
$\mathbf{8 k}$ & $5-\mathrm{Me}$ & $\mathrm{NOH}$ & $69 \%$ \\
$\mathbf{8 1}$ & $\mathrm{H}$ & $\mathrm{NOEt}$ & $51 \%$ \\
$\mathbf{8} \mathbf{8}$ & $5-\mathrm{F}$ & $\mathrm{NOEt}$ & $65 \%$ \\
$\mathbf{8}$ & $5-\mathrm{Me}$ & $\mathrm{NOEt}$ & $69 \%$ \\
\hline
\end{tabular}

Table 2

In vitro activity of gatifloxacin-1,2,3-triazole-isatin hybrids 8a-n against Gram-positive strains

\begin{tabular}{cccccccccc}
\hline \multirow{2}{*}{ Compd. } & \multicolumn{8}{c}{ MIC $(\boldsymbol{\mu g} / \mathbf{m L})$} & \multicolumn{1}{c}{} \\
\cline { 2 - 10 } & S.a. & MSSA & MRSA & S.e. & MSSE & MRSE & S.p. & E.fa & E.fm \\
\hline $\mathbf{8 a}$ & 0.06 & 1 & 2 & 0.5 & 1 & 4 & 0.5 & 1 & 16 \\
$\mathbf{8 b}$ & 0.12 & 0.5 & 1 & 1 & 0.5 & 2 & 0.5 & 2 & 32 \\
$\mathbf{8 c}$ & 0.12 & 0.25 & 1 & 0.5 & 1 & 2 & 0.25 & 1 & 16 \\
$\mathbf{8 d}$ & 1 & 4 & 8 & 4 & 8 & 18 & 2 & 8 & 64 \\
$\mathbf{8 e}$ & 0.25 & 2 & 4 & 1 & 2 & 8 & 1 & 8 & 32 \\
$\mathbf{8 f}$ & 0.25 & 1 & 1 & 0.5 & 1 & 1 & 0.25 & 1 & 16 \\
$\mathbf{8 g}$ & 0.06 & 0.12 & 1 & 0.25 & 0.5 & 1 & 0.12 & 2 & 8 \\
$\mathbf{8 h}$ & 0.5 & 1 & 1 & 0.5 & 4 & 4 & 4 & 8 & 16 \\
$\mathbf{8 i}$ & 0.25 & 0.5 & 1 & 0.5 & 1 & 1 & 0.5 & 1 & 16 \\
$\mathbf{8 j}$ & 0.5 & 1 & 1 & 0.5 & 2 & 4 & 0.5 & 2 & 16 \\
$\mathbf{8 k}$ & 0.25 & 1 & 0.5 & 0.25 & 1 & 2 & 1 & 2 & 16 \\
$\mathbf{8 1}$ & 0.5 & 2 & 4 & 1 & 2 & 2 & 2 & 4 & 32 \\
$\mathbf{8 m}$ & 0.5 & 1 & 4 & 2 & 2 & 4 & 4 & 16 & 32 \\
$\mathbf{8 n}$ & 0.25 & 1 & 2 & 1 & 1 & 2 & 2 & 8 & 16 \\
gatifloxacin & 0.12 & 0.25 & 1 & 0.12 & 0.12 & 0.5 & 0.25 & 1 & 16 \\
vancomycin & 1 & 1 & 1 & 0.5 & 0.5 & 1 & 0.25 & 4 & 1 \\
\hline
\end{tabular}

Abbreviations: S.a., S. aureus; MSSA, methicillin-sensitive S. aureus; MRSA, methicillin-resistant S. aureus; S.e., S. epidermidis; MSSE, methicillin-sensitive S. epidermidis; MRSE, methicillin-resistant $S$. epidermidis; S.p., $S$. pneumoniae; E.fa., E. faecalis; E.fm., E. faecium.

Table 3

In vitro activity of gatifloxacin-1,2,3-triazole-isatin hybrids 8a-n against Gram-negative strains

\begin{tabular}{ccccccccccc}
\hline \multirow{2}{*}{ Compd. } & \multicolumn{10}{c}{ MIC $(\boldsymbol{\mu g} / \mathbf{m L})$} \\
\cline { 2 - 11 } & E.co.1 & E.co.2 & K.p.1 & K.p.2 & P.a. & A.c. & E.c. & E.a. & S.m. & C.f. \\
\hline $\mathbf{8 a}$ & 0.25 & 0.5 & 0.03 & 1 & 1 & 2 & 0.12 & 0.12 & 4 & 2 \\
$\mathbf{8 b}$ & 0.12 & 0.12 & $\leq 0.03$ & 0.5 & 1 & 2 & $\leq 0.03$ & 0.12 & 4 & 1 \\
$\mathbf{8 c}$ & 0.5 & 0.5 & 0.06 & 0.5 & 2 & 1 & $\leq 0.03$ & 0.25 & 8 & 2 \\
$\mathbf{8 d}$ & 1 & 1 & 2 & 0.5 & 16 & 8 & 0.5 & 4 & 16 & 8 \\
$\mathbf{8} \mathbf{8 0}$ & 0.5 & 1 & 0.5 & 1 & 4 & 2 & 1 & 0.5 & 16 & 4 \\
$\mathbf{8 f}$ & 0.5 & 0.5 & $\leq 0.03$ & 1 & 2 & 1 & 0.06 & 0.12 & 16 & 2 \\
$\mathbf{8 g}$ & 0.5 & 0.5 & 0.03 & 1 & 4 & 2 & 0.25 & 0.25 & 8 & 4 \\
$\mathbf{8 h}$ & 1 & 2 & 0.25 & 2 & 2 & 1 & 0.5 & 1 & 16 & 8 \\
$\mathbf{8 i}$ & 4 & 4 & 0.5 & 0.25 & 4 & 2 & 1 & 1 & 32 & 8 \\
$\mathbf{8 j}$ & 2 & 2 & 0.5 & 0.25 & 2 & 2 & 1 & 0.5 & 8 & 16 \\
$\mathbf{8 k}$ & 2 & 4 & 0.25 & 0.5 & 2 & 4 & 0.5 & 1 & 8 & 16 \\
$\mathbf{8 1}$ & 1 & 0.5 & 0.12 & 1 & 0.5 & 2 & 0.12 & 0.25 & 8 & 1 \\
$\mathbf{8 m}$ & 1 & 0.5 & 0.25 & 1 & 2 & 4 & 0.25 & 0.5 & 8 & 2 \\
$\mathbf{8 n}$ & 2 & 1 & 0.12 & 2 & 1 & 4 & 0.12 & 0.5 & 4 & 4 \\
gatifloxacin & 0.12 & 0.12 & 0.06 & 0.5 & 0.5 & 1 & $\leq 0.03$ & 0.25 & 8 & 1 \\
vancomycin & $>128$ & $>128$ & $>128$ & $>128$ & $>128$ & $>128$ & $>128$ & $>128$ & $>128$ & $>128$ \\
\hline
\end{tabular}

Abbreviations: E.co.1, Escherichia coli ESBLs(-); E.co.2, Escherichia coli ESBLs(+); K.p.1, Klebsiella pneumoniae ESBLs(+); K.p.2, Klebsiella pneumonia ESBLs(-); P.a., Pseudomonas aeruginosa; A.c., Acinetobactercal coacetious; E.c., Enterobacter cloacae; E.a., Enterobacter aerogenes; S.m., S. maltophilia; C.f., C. freundii; ESBLs(+): Extended spectrum beta-lactamases (ESBLs).

The antibacterial activity of the methylene and acetyl tethered gatifloxacin-1,2,3-triazole-isatin hybrids 8a-n against a panel of Gram-positive and Gram-negative bacteria including drug-resistant 
strains was investigated, and the minimum inhibitory concentration (MIC) values were presented in Table 2 and 3.

It can be seen from Table 2, all hybrids 8a-n exhibited promising activity against $S$. aureus, MSSA, MRSA, $S$. epidermidis, MSSE, MRSE, $S$. pneumoniae, E. faecalis, and E. faecium with MIC values ranging from 0.06 to $32 \mu \mathrm{g} / \mathrm{mL}$, and some of them were comparable to or better than the parent gatifloxacin (MIC: $0.12-16 \mu \mathrm{g} / \mathrm{mL}$ ) and reference vancomycin (MIC: $0.25-4 \mu \mathrm{g} / \mathrm{mL}$ ) against certain strains. The structure-activity relationship (SAR) revealed that introduction of methyl group at C-5 position of isatin moiety could enhance the activity greatly, while fluoro has little influence on the activity. Shift the fluoro to C-7 position or incorporation of chloro at both $\mathrm{C}-5$ and $\mathrm{C}-7$ positions was harmful to the activity as evidenced by that hybrids $\mathbf{8 d}$ and 8e showed the lowest activity in the series, suggesting that introduction of substituents at C-7 position of isatin motif may interfere the interaction between the hybrid molecular and action target. Replacement of ketone by methyloxime ( $\mathrm{R}_{2}$ position) at $\mathrm{C}-3$ position of isatin motif was favorable to the activity, while ethyloxime and oxime could not increase the activity, and the relative contribution order was methyloxime $>$ ketone $\approx$ oxime $>$ ethyloxime. The most active hybrid 8g (MIC: 0.06-8 $\mu \mathrm{g} / \mathrm{mL}$ ) was no inferior to the parent gatifloxacin (MIC: $0.06-8 \mu \mathrm{g} / \mathrm{mL}$ ) against the tested Grampositive pathogens and was 2-16 times more potent than gatifloxacin and vancomycin against $S$. aureus, MSSA, and S. pneumoniae strains.

From the data presented in Table 3, it can be concluded that all hybrids 8a-n (MIC: $\leq 0.03$ $32 \mu \mathrm{g} / \mathrm{mL}$ ) showed considerable activity against $E$. coli $\operatorname{ESBLs}(-), E$. coli $\mathrm{ESBLs}(+), K$. pneumoniae ESBLs(+), K. pneumonia ESBLs(-), A. coacetious, $E$. cloacae, E. aerogenes, S. maltophilia; and $C$. freundii. The antibacterial activity of 8a-g was no inferior to the parent gatifloxacin (MIC: $\leq 0.03-8$ $\mu \mathrm{g} / \mathrm{mL}$ ) and was far more potent than vancomycin (MIC: $>128 \mu \mathrm{g} / \mathrm{mL}$ ) against all tested Gram-negative bacteria. The SAR revealed that replacement of ketone at C-3 position of isatin fragment by methyloxime, ethyloxime and oxime was disfavorable to the activity, and the contribution order was ketone $\geq$ methyloxime $>$ ethyloxime $>$ oxime. Hybrids with fluoro at C-5 position exhibited higher activity, while hybrids with 5-methyl, 7-fluoro and 5,7-dichloro showed lower activity when compared to unsubstituted analogs, suggesting the substituents at C-5 and C-7 positions have great influence on the interaction between the hybrid molecular and bonding sites.
Among them, the most potent hybrid 8b (MIC: $\leq 0.03-4 \mu \mathrm{g} / \mathrm{mL}$ ) was comparable to or superior to the parent gatifloxacin against the tested Gram-positive strains.

\section{Table 4}

In vitro cytotoxicity of methylene and acetyl tethered gatifloxacin-1,2,3-triazole-isatin hybrids 8a-n towards VERO cells

\begin{tabular}{|c|c|}
\hline Compd. & $\mathrm{CC}_{50}(\mu \mathrm{g} / \mathrm{mL})$ \\
\hline $8 \mathbf{a}$ & 32 \\
\hline $8 b$ & 32 \\
\hline $8 \mathrm{c}$ & 128 \\
\hline $8 d$ & 16 \\
\hline $8 e$ & 64 \\
\hline $8 f$ & 64 \\
\hline $8 g$ & 16 \\
\hline $8 h$ & 32 \\
\hline $8 \mathbf{i}$ & 128 \\
\hline $8 \mathbf{j}$ & 16 \\
\hline $8 \mathbf{k}$ & 32 \\
\hline 81 & 128 \\
\hline $8 m$ & 16 \\
\hline $8 n$ & 64 \\
\hline gatifloxacin & $>128$ \\
\hline
\end{tabular}

From Table 4, all hybrids 8a-n also displayed acceptable cytotoxicity towards VERO cells with half-cytotoxic concentration $\left(\mathrm{CC}_{50}\right)$ in a range of 16 to $128 \mu \mathrm{g} / \mathrm{mL}$, but they were more toxic than the parent gatifloxacin $\left(\mathrm{CC}_{50}:>128 \mu \mathrm{g} / \mathrm{mL}\right)$. The structure-cytotoxicity relationship suggested that incorporation of oxime, ethyloxime or methyloxime at $\mathrm{C}-3$ position or introduction of either electron-donating or electron-withdrawing groups at C-5 or C-7 position of isatin motif could increase the cytotoxicity when compared with unsubstituted analogs. 
Among them, hybrids $\mathbf{8 b}\left(\mathrm{CC}_{50}: 32 \mu \mathrm{g} / \mathrm{mL}\right)$ and $\mathbf{8 g}\left(\mathrm{CC}_{50}: 16 \mu \mathrm{g} / \mathrm{mL}\right)$ with highest activity against Gram-positive and Gram-negative pathogens respectively, also demonstrated acceptable cytotoxicity towards VERO cells. Thus, these two hybrids could serve as lead compounds for further investigations.

\section{EXPERIMENTAL}

\section{Synthesis}

To a suspension of gatifloxacin $(50 \mathrm{mmol})$ in $N, N$ dimethylformamide (DMF, $500 \mathrm{~mL})$, NHS ester 6 (60 mmol), DIPEA $(100 \mathrm{~mL})$ in tetrahydrofuran (THF, $100 \mathrm{~mL}$ ) was added during a period of $10 \mathrm{~min}$ under nitrogen atmosphere at $0{ }^{\circ} \mathrm{C}$. The mixture was stirred at room temperature overnight, and then the solvent was evaporated under reduced pressure. The residue was purified by silica gel chromatography eluted with dichloromethane $(\mathrm{DCM})$ : methanol $(\mathrm{MeOH})=10: 1$ to give the gatifloxacin intermediate 7 .

To a mixture of gatifloxacin derivative $7(6 \mathrm{mmol})$ and isatin intermediates 2 or $3(8 \mathrm{mmol})$ in DMF $(60 \mathrm{~mL})$, $\mathrm{Cu}(\mathrm{OAc})_{2}(1 \mathrm{mmol})$ was added. The mixture was stirred at 40 ${ }^{\circ} \mathrm{C}$ for $8 \mathrm{~h}$, and the filtered. The filtrate was concentrated under reduced pressure, and the residue was purified by reverse phase column with formic acid as additive to give the desired products 8 .

1.1. 1-cyclopropyl-7-(4-(2-(4-((2,3-dioxoindolin-1yl)methyl)-1H-1,2,3-triazol-1-yl)acetyl)-3methylpiperazin-1-yl)-6-fluoro-8-methoxy-4-oxo1,4-dihydroquinoline-3-carboxylic acid (8a)

Yellow solid, yield: $63 \%$. ${ }^{1} \mathrm{H}$ NMR $\left(400 \mathrm{MHz}\right.$, DMSO- $\left.d_{6}\right)$ $\delta 1.03-1.43\left(7 \mathrm{H}, \mathrm{m}\right.$, cyclopropyl-4H and $\left.-\mathrm{CH}_{3}\right), 3.15-3.56(5 \mathrm{H}$, $\mathrm{m}$, piperazinyl-5H), 3.74-4.26 $(6 \mathrm{H}, \mathrm{m}$, piperazinyl-2 $\mathrm{H}$, cyclopropyl- $1 \mathrm{H}$ and $\left.-\mathrm{OCH}_{3}\right), 5.01\left(2 \mathrm{H}, \mathrm{s},-\mathrm{CH}_{2}\right.$ - linker), 5.53$5.65\left(2 \mathrm{H}, \mathrm{m},-\mathrm{CH}_{2}-\right.$ linker $), 7.15(1 \mathrm{H}, \mathrm{t}, J=8.0 \mathrm{~Hz}, \mathrm{Ar}-\mathrm{H})$, $7.20(1 \mathrm{H}, \mathrm{d}, J=8.0 \mathrm{~Hz}, \mathrm{Ar}-\mathrm{H}), 7.59(1 \mathrm{H}, \mathrm{d}, J=8.0 \mathrm{~Hz}, \mathrm{Ar}-\mathrm{H})$, $7.66(1 \mathrm{H}, \mathrm{t}, J=8.0 \mathrm{~Hz}, \mathrm{Ar}-\mathrm{H}), 7.80(1 \mathrm{H}, \mathrm{d}, J=8.0 \mathrm{~Hz}, \mathrm{Ar}-\mathrm{H})$, $8.11(1 \mathrm{H}, \mathrm{s}, \mathrm{Ar}-\mathrm{H}), 8.72(1 \mathrm{H}, \mathrm{s}, \operatorname{Ar}-\mathrm{H}), 14.92(1 \mathrm{H}, \mathrm{brs}$, $\mathrm{COOH}$. HRMS-ESI: $\mathrm{m} / \mathrm{z}$ Calcd. for $\mathrm{C}_{32} \mathrm{H}_{31} \mathrm{FN}_{7} \mathrm{O}_{7}[\mathrm{M}+\mathrm{H}]^{+}$: 644.22635; Found: 644.22407.

\subsection{1-cyclopropyl-6-fluoro-7-(4-(2-(4-)((5-fluoro-2,3-} dioxoindolin-1-yl)methyl)-1H-1,2,3-triazol-1yl)acetyl)-3-methylpiperazin-1-yl)-8-methoxy-4-oxo1,4-dihydroquinoline-3-carboxylic acid $\mathbf{( 8 \mathbf { b } )}$

Yellow solid, yield: 59\%. ${ }^{1} \mathrm{H}$ NMR $\left(400 \mathrm{MHz}\right.$, DMSO- $\left.d_{6}\right)$ $\delta$ 1.01-1.42 $\left(7 \mathrm{H}, \mathrm{m}\right.$, cyclopropyl-4H and $\left.-\mathrm{CH}_{3}\right), 3.17-3.55(5 \mathrm{H}$, $\mathrm{m}$, piperazinyl $-5 \mathrm{H}), \quad 3.73-4.26(6 \mathrm{H}, \mathrm{m}$, piperazinyl- $2 \mathrm{H}$, cyclopropyl-1H and $\left.-\mathrm{OCH}_{3}\right), 5.01\left(2 \mathrm{H}, \mathrm{s},-\mathrm{CH}_{2}\right.$ - linker $), 5.43-$ $5.65\left(2 \mathrm{H}, \mathrm{m},-\mathrm{CH}_{2}-\right.$ linker $), 7.22(1 \mathrm{H}, \mathrm{d}, J=4.0 \mathrm{~Hz}, \mathrm{Ar}-\mathrm{H})$, 7.48-7.56 (2H, m, Ar-H), $7.76(1 \mathrm{H}, \mathrm{d}, J=12.0 \mathrm{~Hz}, \mathrm{Ar}-\mathrm{H}), 8.11$ $(1 \mathrm{H}, \mathrm{s}, \mathrm{Ar}-\mathrm{H}), 8.71(1 \mathrm{H}, \mathrm{s}, \mathrm{Ar}-\mathrm{H}), 14.94(1 \mathrm{H}$, brs, $\mathrm{COOH})$. HRMS-ESI: $\mathrm{m} / \mathrm{z}$ Calcd. for $\mathrm{C}_{32} \mathrm{H}_{30} \mathrm{~F}_{2} \mathrm{~N}_{7} \mathrm{O}_{7} \quad[\mathrm{M}+\mathrm{H}]^{+}$: 662.21693; Found: 662.21518 .

\subsection{1-cyclopropyl-6-fluoro-8-methoxy-7-(3-methyl-4-(2-} (4-((5-methyl-2,3-dioxoindolin-1-yl)methyl)-1H1,2,3-triazol-1-yl)acetyl)piperazin-1-yl)-4-oxo-1,4dihydroquinoline-3-carboxylic acid $\mathbf{( 8 c )}$

Yellow solid, yield: $71 \% .{ }^{1} \mathrm{H}$ NMR $\left(400 \mathrm{MHz}, \mathrm{CDCl}_{3}\right) \delta$ 1.01-1.52 $\left(7 \mathrm{H}, \mathrm{m}\right.$, cyclopropyl-4H and $\left.-\mathrm{CH}_{3}\right), 2.32(3 \mathrm{H}, \mathrm{s}$, $\left.-\mathrm{CH}_{3}\right), 3.26-3.55(6 \mathrm{H}, \mathrm{m}$, piperazinyl-6H), 3.71-3.75 $(4 \mathrm{H}, \mathrm{m}$, piperazinyl- $1 \mathrm{H}$, and $\left.-\mathrm{OCH}_{3}\right), 4.01-4.02(1 \mathrm{H}, \mathrm{m}$, cyclopropyl$1 \mathrm{H}), 5.02\left(2 \mathrm{H}, \mathrm{s},-\mathrm{CH}_{2}\right.$ - linker $), 5.22-5.39\left(2 \mathrm{H}, \mathrm{m},-\mathrm{CH}_{2}-\right.$ linker), $7.16(1 \mathrm{H}, \mathrm{d}, J=8.0 \mathrm{~Hz}, \mathrm{Ar}-\mathrm{H}), 7.38-7.40(2 \mathrm{H}, \mathrm{m}, \mathrm{Ar}-$ H), 7.83-7.86 (2H, m, Ar-H), $8.82(1 \mathrm{H}, \mathrm{s}, \mathrm{Ar}-\mathrm{H})$.

1.4. 1-cyclopropyl-6-fluoro-7-(4-(2-(4-((7-fluoro-2,3dioxoindolin-1-yl)methyl)-1H-1,2,3-triazol-1yl)acetyl)-3-methylpiperazin-1-yl)-8-methoxy-4-oxo1,4-dihydroquinoline-3-carboxylic acid (8d)

Yellow solid, yield: $43 \%$. ${ }^{1} \mathrm{H}$ NMR $\left(400 \mathrm{MHz}, \mathrm{CDCl}_{3}\right) \delta$ 1.01-1.38 $\left(7 \mathrm{H}, \mathrm{m}\right.$, cyclopropyl-4H and $\left.-\mathrm{CH}_{3}\right), 3.30-3.57(4 \mathrm{H}$, $\mathrm{m}$, piperazinyl-4H), $3.76\left(3 \mathrm{H}, \mathrm{s},-\mathrm{OCH}_{3}\right), 4.03-4.04(1 \mathrm{H}, \mathrm{m}$, cyclopropyl-1H), 4.47-4.82 (3H, m, piperazinyl-3H), 5.23 $\left(2 \mathrm{H}, \mathrm{s},-\mathrm{CH}_{2}-\right.$ linker $), 5.32-5.39\left(2 \mathrm{H}, \mathrm{m},-\mathrm{CH}_{2}-\right.$ linker $), 7.12$ $(1 \mathrm{H}, \mathrm{t}, J=4.0 \mathrm{~Hz}, \mathrm{Ar}-\mathrm{H}), 7.34-7.39(1 \mathrm{H}, \mathrm{m}, \mathrm{Ar}-\mathrm{H}), 7.44(1 \mathrm{H}$, d, $J=8.0 \mathrm{~Hz}, \mathrm{Ar}-\mathrm{H}), 7.87-7.92(2 \mathrm{H}, \mathrm{m}, \mathrm{Ar}-\mathrm{H}), 8.82(1 \mathrm{H}, \mathrm{s}, \mathrm{Ar}-$ $\mathrm{H}), 14.82(1 \mathrm{H}$, brs, COOH). HRMS-ESI: $\mathrm{m} / \mathrm{z}$ Calcd. for $\mathrm{C}_{32} \mathrm{H}_{30} \mathrm{~F}_{2} \mathrm{~N}_{7} \mathrm{O}_{7}[\mathrm{M}+\mathrm{H}]^{+}$: 662.21693; Found: 662.21447.

\subsection{1-cyclopropyl-7-(4-(2-(4-((5,7-dichloro-2,3-} dioxoindolin-1-yl)methyl)-1H-1,2,3-triazol-1yl)acetyl)-3-methylpiperazin-1-yl)-6-fluoro-8methoxy-4-oxo-1,4-dihydroquinoline-3-carboxylic acid $(\mathbf{8 e})$

Yellow solid, yield: $22 \% .{ }^{1} \mathrm{H}$ NMR (400 MHz, DMSO- $d_{6}$ ) $\delta$ 1.02-1.42 $\left(7 \mathrm{H}, \mathrm{m}\right.$, cyclopropyl-4H and $\left.-\mathrm{CH}_{3}\right), 3.14-3.55(6 \mathrm{H}$, $\mathrm{m}$, piperazinyl-6H), 3.73-3.81 $(4 \mathrm{H}, \mathrm{m}$, piperazinyl-1 $\mathrm{H}$ and $\left.\mathrm{OCH}_{3}\right), 4.15-4.16(1 \mathrm{H}, \mathrm{m}$, cyclopropyl- $1 \mathrm{H}), 5.29\left(2 \mathrm{H}, \mathrm{s},-\mathrm{CH}_{2}-\right.$ linker), 5.43-5.69 (2H, m, $-\mathrm{CH}_{2}$ - linker), $7.20(1 \mathrm{H}, \mathrm{d}, J=8.0$ $\mathrm{Hz}, \mathrm{Ar}-\mathrm{H}), 7.64(1 \mathrm{H}, \mathrm{d}, J=12.0 \mathrm{~Hz}, \mathrm{Ar}-\mathrm{H}), 7.76(1 \mathrm{H}, \mathrm{d}$, $J=12.0 \mathrm{~Hz}, \operatorname{Ar}-\mathrm{H}), 8.12(1 \mathrm{H}, \mathrm{s}, \operatorname{Ar}-\mathrm{H}), 8.72(1 \mathrm{H}, \mathrm{s}, \operatorname{Ar}-\mathrm{H})$, $14.91(1 \mathrm{H}$, brs, $\mathrm{COOH})$. HRMS-ESI: $\mathrm{m} / \mathrm{z}$ Calcd. for $\mathrm{C}_{32} \mathrm{H}_{29} \mathrm{Cl}_{2} \mathrm{FN}_{7} \mathrm{O}_{7}[\mathrm{M}+\mathrm{H}]^{+}:$712.14841; Found: 712.14639.

\subsection{1-cyclopropyl-6-fluoro-8-methoxy-7-(4-(2-(4-)((3-} (methoxyimino)-2-oxoindolin-1-yl)methyl)-1H-1,2,3triazol-1-yl)acetyl)-3-methylpiperazin-1-yl)-4-oxo1,4-dihydroquinoline-3-carboxylic acid ( $\mathbf{8 f})$

Yellow solid, yield: $79 \%$. ${ }^{1} \mathrm{H}$ NMR $\left(400 \mathrm{MHz}, \mathrm{CDCl}_{3}\right.$ ) $\delta$ 1.01-1.49 $\left(7 \mathrm{H}, \mathrm{m}\right.$, cyclopropyl-4H and $\left.-\mathrm{CH}_{3}\right), 3.29-3.55(5 \mathrm{H}$, $\mathrm{m}$, piperazinyl-5H), 3.74-3.76 $(4 \mathrm{H}, \mathrm{m}$, piperazinyl- $1 \mathrm{H}$ and $\left.-\mathrm{OCH}_{3}\right), 4.02-4.03(1 \mathrm{H}, \mathrm{m}$, cyclopropyl-1H), 4.27-4.46 $(4 \mathrm{H}$, $\mathrm{m}$, piperazinyl-1H and $\left.-\mathrm{NOCH}_{3}\right), 5.09\left(2 \mathrm{H}, \mathrm{s},-\mathrm{CH}_{2}-\right.$ linker $)$, 5.19-5.32 $\left(2 \mathrm{H}, \mathrm{m},-\mathrm{CH}_{2}\right.$ - linker), $7.06(1 \mathrm{H}, \mathrm{t}, J=8.0 \mathrm{~Hz}, \mathrm{Ar}-$ H), $7.20(1 \mathrm{H}, \mathrm{d}, J=8.0 \mathrm{~Hz}, \mathrm{Ar}-\mathrm{H}), 7.36(1 \mathrm{H}, \mathrm{d}, J=8.0 \mathrm{~Hz}, \mathrm{Ar}-$ $\mathrm{H}), 7.81(1 \mathrm{H}, \mathrm{d}, J=8.0 \mathrm{~Hz}, \mathrm{Ar}-\mathrm{H}), 7.90-7.95(2 \mathrm{H}, \mathrm{m}, \mathrm{Ar}-\mathrm{H})$, $8.84(1 \mathrm{H}, \mathrm{s}, \mathrm{Ar}-\mathrm{H})$. HRMS-ESI: $\mathrm{m} / \mathrm{z}$ Calcd. for $\mathrm{C}_{33} \mathrm{H}_{34} \mathrm{FN}_{8} \mathrm{O}_{7}$ $[\mathrm{M}+\mathrm{H}]^{+}:$673.25290; Found: 673.24989.

\subsection{1-cyclopropyl-6-fluoro-7-(4-(2-(4-((5-fluoro-2,3-} dioxoindolin-1-yl)methyl)-1H-1,2,3-triazol-1yl)acetyl)-3-methylpiperazin-1-yl)-8-methoxy-4-oxo1,4-dihydroquinoline-3-carboxylic acid $\mathbf{( 8 g})$

Yellow solid, yield: $68 \% .{ }^{1} \mathrm{H}$ NMR $\left(400 \mathrm{MHz}, \mathrm{CDCl}_{3}\right)$ $\delta$ 1.02-1.38 $\left(7 \mathrm{H}, \mathrm{m}\right.$, cyclopropyl-4H and $\left.-\mathrm{CH}_{3}\right), 3.29-3.54(5 \mathrm{H}$, $\mathrm{m}$, piperazinyl-5H), 3.73-3.77 $(4 \mathrm{H}, \mathrm{m}$, piperazinyl-1 $\mathrm{H}$ and $\left.-\mathrm{OCH}_{3}\right), 4.02-4.03(1 \mathrm{H}, \mathrm{m}$, cyclopropyl-1H), 4.28-4.32 $(4 \mathrm{H}$, $\mathrm{m}$, piperazinyl- $1 \mathrm{H}$ and $\left.-\mathrm{NOCH}_{3}\right), 5.08\left(2 \mathrm{H}, \mathrm{s},-\mathrm{CH}_{2}\right.$ - linker), 5.20-5.32 (2H, m, $-\mathrm{CH}_{2}$ - linker), 7.08-7.20 (2H, m, Ar-H), $7.68(1 \mathrm{H}, \mathrm{d}, J=4.0 \mathrm{~Hz}, \mathrm{Ar}-\mathrm{H}), 7.82(1 \mathrm{H}, \mathrm{s}, \mathrm{Ar}-\mathrm{H}), 7.92(1 \mathrm{H}$, $\mathrm{d}, J=12.0 \mathrm{~Hz}, \mathrm{Ar}-\mathrm{H}), 8.85(1 \mathrm{H}, \mathrm{s}, \operatorname{Ar}-\mathrm{H}), 14.65(1 \mathrm{H}, \mathrm{brs}$, COOH). HRMS-ESI: m/z Calcd. for $\mathrm{C}_{33} \mathrm{H}_{33} \mathrm{~F}_{2} \mathrm{~N}_{8} \mathrm{O}_{7}[\mathrm{M}+\mathrm{H}]^{+}$: 691.24348; Found: 691.24071.

1.8. 1-cyclopropyl-6-fluoro-7-(4-(2-(4-((7-fluoro-3(methoxyimino)-2-oxoindolin-1-yl)methyl)-1H-1,2,3triazol-1-yl)acetyl)-3-methylpiperazin-1-yl)-8methoxy-4-oxo-1,4-dihydroquinoline-3-carboxylic acid $\mathbf{( 8 h})$

Yellow solid, yield: $57 \%$. ${ }^{1} \mathrm{H}$ NMR (400 MHz, DMSO- $\left.d_{6}\right)$ $\delta$ 0.82-1.43 $\left(7 \mathrm{H}, \mathrm{m}\right.$, cyclopropyl-4H and $\left.-\mathrm{CH}_{3}\right), 3.13-3.24(5 \mathrm{H}$, $\mathrm{m}$, piperazinyl-5H), 3.70-3.79 $(4 \mathrm{H}, \mathrm{m}$, piperazinyl- $1 \mathrm{H}$ and 
$\left.-\mathrm{OCH}_{3}\right), 3.98-3.99(1 \mathrm{H}, \mathrm{m}$, cyclopropyl-1H), 4.19-4.26 (4H, $\mathrm{m}$, piperazinyl- $1 \mathrm{H}$ and $\left.-\mathrm{NOCH}_{3}\right), 5.09\left(2 \mathrm{H}, \mathrm{s},-\mathrm{CH}_{2}-\right.$ linker), 5.40-5.63 (2H, m, - $\mathrm{CH}_{2}$ - linker), 7.10-7.15 (1H, m, Ar-H), $7.38(1 \mathrm{H}, \mathrm{t}, J=8.0 \mathrm{~Hz}, \mathrm{Ar}-\mathrm{H}), 7.66(1 \mathrm{H}, \mathrm{d}, J=12.0 \mathrm{~Hz}, \mathrm{Ar}-$ $\mathrm{H}), 7.78(1 \mathrm{H}, \mathrm{t}, J=8.0 \mathrm{~Hz}, \mathrm{Ar}-\mathrm{H}), 8.02(1 \mathrm{H}, \mathrm{s}, \mathrm{Ar}-\mathrm{H}), 8.64$ $\left(1 \mathrm{H}, \mathrm{s}\right.$, Ar-H). HRMS-ESI: $\mathrm{m} / \mathrm{z}$ Calcd. for $\mathrm{C}_{33} \mathrm{H}_{33} \mathrm{~F}_{2} \mathrm{~N}_{8} \mathrm{O}_{7}$ $[\mathrm{M}+\mathrm{H}]^{+}:$691.24348; Found: 691.24052.

\subsection{1-cyclopropyl-6-fluoro-7-(4-(2-(4-((3-} (hydroxyimino)-2-oxoindolin-1-yl)methyl)-1H-1,2,3triazol-1-yl)acetyl)-3-methylpiperazin-1-yl)-8methoxy-4-oxo-1,4-dihydroquinoline-3-carboxylic acid (8i)

Yellow solid, yield: $47 \% .{ }^{1} \mathrm{H}$ NMR (400 MHz, DMSO- $d_{6}$ ) $\delta 1.00-1.42\left(7 \mathrm{H}, \mathrm{m}\right.$, cyclopropyl-4H and $\left.-\mathrm{CH}_{3}\right), 3.16-3.55(5 \mathrm{H}$, $\mathrm{m}$, piperazinyl-5H), 3.73-3.81 $(4 \mathrm{H}, \mathrm{m}$, piperazinyl-1H and $\left.-\mathrm{OCH}_{3}\right), 4.15-4.25(2 \mathrm{H}, \mathrm{m}$, piperazinyl-1H and cyclopropyl$1 \mathrm{H}), 5.03\left(2 \mathrm{H}, \mathrm{s},-\mathrm{CH}_{2}\right.$ - linker $), 5.76\left(2 \mathrm{H}, \mathrm{s},-\mathrm{CH}_{2}\right.$ - linker), $7.09(1 \mathrm{H}, \mathrm{t}, J=8.0 \mathrm{~Hz}, \mathrm{Ar}-\mathrm{H}), 7.18(1 \mathrm{H}, \mathrm{d}, J=8.0 \mathrm{~Hz}, \mathrm{Ar}-\mathrm{H})$, $7.42(1 \mathrm{H}, \mathrm{t}, J=8.0 \mathrm{~Hz}, \mathrm{Ar}-\mathrm{H}), 7.76(1 \mathrm{H}, \mathrm{d}, J=12.0 \mathrm{~Hz}, \mathrm{Ar}-$ $\mathrm{H}), 8.02(1 \mathrm{H}, \mathrm{s}, \mathrm{Ar}-\mathrm{H}), 8.32(1 \mathrm{H}, \mathrm{s}, \mathrm{Ar}-\mathrm{H}), 8.74(1 \mathrm{H}, \mathrm{s}, \mathrm{Ar}-\mathrm{H})$. HRMS-ESI: $\mathrm{m} / \mathrm{z}$ Calcd. for $\mathrm{C}_{32} \mathrm{H}_{32} \mathrm{FN}_{8} \mathrm{O}_{7}[\mathrm{M}+\mathrm{H}]^{+}: 659.23725$; Found: 659.23402.

1.10. 1-cyclopropyl-6-fluoro-7-(4-(2-(4-((5-fluoro-2,3dioxoindolin-1-yl)methyl)-1H-1,2,3-triazol-1yl)acetyl)-3-methylpiperazin-1-yl)-8-methoxy-4-oxo1,4-dihydroquinoline-3-carboxylic acid $\mathbf{( 8 \mathbf { j } )}$

Yellow solid, yield: $56 \%$. ${ }^{1} \mathrm{H}$ NMR $\left(400 \mathrm{MHz}, \mathrm{CDCl}_{3}\right) \delta$ 1.02-1.47 $\left(7 \mathrm{H}, \mathrm{m}\right.$, cyclopropyl-4H and $\left.-\mathrm{CH}_{3}\right), 3.28-3.57(5 \mathrm{H}$, $\mathrm{m}$, piperazinyl-5H), 3.73-3.75 $(4 \mathrm{H}, \mathrm{m}$, piperazinyl- $1 \mathrm{H}$ and $\left.-\mathrm{OCH}_{3}\right), 4.02-4.04(1 \mathrm{H}, \mathrm{m}$, cyclopropyl-1H), 4.14-4.16 $(1 \mathrm{H}$, $\mathrm{m}$, piperazinyl-1H), $5.05\left(2 \mathrm{H}, \mathrm{d}, J=12.0 \mathrm{~Hz},-\mathrm{CH}_{2}\right.$ - linker $)$, 5.21-5.35 (2H, m, $-\mathrm{CH}_{2}$ - linker), 6.97-7.14 $(3 \mathrm{H}, \mathrm{m}, \mathrm{Ar}-\mathrm{H})$, 7.79-7.95 (2H, m, Ar-H), $8.85(1 \mathrm{H}, \mathrm{s}, \mathrm{Ar}-\mathrm{H}), 14.68$ (1H, brs, COOH). HRMS-ESI: $\mathrm{m} / \mathrm{z}$ Calcd. for $\mathrm{C}_{32} \mathrm{H}_{31} \mathrm{~F}_{2} \mathrm{~N}_{8} \mathrm{O}_{7}[\mathrm{M}+\mathrm{H}]^{+}$: 677.22783; Found: 677.22512.

\subsection{1-cyclopropyl-6-fluoro-7-(4-(2-(4-((3-} (hydroxyimino)-5-methyl-2-oxoindolin-1-yl)methyl)1H-1,2,3-triazol-1-yl)acetyl)-3-methylpiperazin-1yl)-8-methoxy-4-oxo-1,4-dihydroquinoline-3carboxylic acid $\mathbf{( 8 k})$

Yellow solid, yield: $69 \%$. ${ }^{1} \mathrm{H}$ NMR $\left(400 \mathrm{MHz}, \mathrm{DMSO}-d_{6}\right)$ $\delta$ 1.03-1.41 $\left(7 \mathrm{H}, \mathrm{m}\right.$, cyclopropyl- $4 \mathrm{H}$ and $\left.-\mathrm{CH}_{3}\right), 2.26(3 \mathrm{H}, \mathrm{s}$, $\left.-\mathrm{CH}_{3}\right), 3.17-3.55\left(6 \mathrm{H}, \mathrm{m}\right.$, piperazinyl-6H), $3.73\left(3 \mathrm{H}, \mathrm{s},-\mathrm{OCH}_{3}\right)$, 4.16-4.18 $(2 \mathrm{H}, \mathrm{m}$, piperazinyl-1H and cyclopropyl-1H), 5.00 (2H, s, - $\mathrm{CH}_{2}$ - linker), 5.38-5.67 (2H, m, $-\mathrm{CH}_{2}$ - linker), 7.04 $(1 \mathrm{H}, \mathrm{d}, J=8.0 \mathrm{~Hz}, \mathrm{Ar}-\mathrm{H}), 7.24(1 \mathrm{H}, \mathrm{d}, J=4.0 \mathrm{~Hz}, \mathrm{Ar}-\mathrm{H}), 7.80$ $(1 \mathrm{H}, \mathrm{d}, J=12.0 \mathrm{~Hz}, \mathrm{Ar}-\mathrm{H}), 8.00(1 \mathrm{H}, \mathrm{s}, \mathrm{Ar}-\mathrm{H}), 8.73(1 \mathrm{H}, \mathrm{s}, \mathrm{Ar}-$ $\mathrm{H}), 14.92(1 \mathrm{H}$, brs, COOH). HRMS-ESI: $\mathrm{m} / \mathrm{z}$ Calcd. for $\mathrm{C}_{33} \mathrm{H}_{34} \mathrm{FN}_{8} \mathrm{O}_{7}[\mathrm{M}+\mathrm{H}]^{+}$: 673.25290; Found: 673.25076 .

1.12. 1-cyclopropyl-7-(4-(2-(4-((3-(ethoxyimino)-2oxoindolin-1-yl)methyl)-1H-1,2,3-triazol-1yl)acetyl)-3-methylpiperazin-1-yl)-6-fluoro-8methoxy-4-oxo-1,4-dihydroquinoline-3-carboxylic acid (81)

Yellow solid, yield: $51 \% .{ }^{1} \mathrm{H}$ NMR $\left(400 \mathrm{MHz}\right.$, DMSO- $\left.d_{6}\right)$ $\delta$ 1.20-1.41 (10H, m, $\mathrm{NOCH}_{2} \mathrm{CH}_{3}$, cyclopropyl-4H and $\left.-\mathrm{CH}_{3}\right)$, 3.34-3.42 $(4 \mathrm{H}, \mathrm{m}$, piperazinyl- $4 \mathrm{H}), 3.70-3.82\left(6 \mathrm{H}, \mathrm{m},-\mathrm{OCH}_{3}\right.$ and piperazinyl-3H) $4.45-4.58\left(3 \mathrm{H}, \mathrm{m}, \mathrm{NOCH}_{2} \mathrm{CH}_{3}\right.$ and cyclopropyl-1H), $5.02\left(2 \mathrm{H}, \mathrm{s},-\mathrm{CH}_{2}-\right.$ linker $), 5.52(2 \mathrm{H}, \mathrm{s}$, $-\mathrm{CH}_{2}$ - linker), $7.10(1 \mathrm{H}, \mathrm{t}, J=8.0 \mathrm{~Hz}, \mathrm{Ar}-\mathrm{H}), 7.19(1 \mathrm{H}, \mathrm{d}$, $J=4.0 \mathrm{~Hz}, \mathrm{Ar}-\mathrm{H}), 7.46(1 \mathrm{H}, \mathrm{d}, J=8.0 \mathrm{~Hz}, \mathrm{Ar}-\mathrm{H}), 7.90-7.95$ $(2 \mathrm{H}, \mathrm{m}, \mathrm{Ar}-\mathrm{H}), 8.03(1 \mathrm{H}, \mathrm{s}, \mathrm{Ar}-\mathrm{H}), 8.68(1 \mathrm{H}, \mathrm{s}, \mathrm{Ar}-\mathrm{H})$. HRMSESI: $\mathrm{m} / \mathrm{z}$ Calcd. for $\mathrm{C}_{34} \mathrm{H}_{36} \mathrm{FN}_{8} \mathrm{O}_{7}[\mathrm{M}+\mathrm{H}]^{+}:$: 687.26855; Found: 687.26346
1.13. 1-cyclopropyl-6-fluoro-7-(4-(2-(4-((5-fluoro-2,3dioxoindolin-1-yl)methyl)-1H-1,2,3-triazol-1yl)acetyl)-3-methylpiperazin-1-yl)-8-methoxy-4-oxo1,4-dihydroquinoline-3-carboxylic acid $\mathbf{( 8 m})$

Yellow solid, yield: $65 \%$. ${ }^{1} \mathrm{H}$ NMR $\left(400 \mathrm{MHz}, \mathrm{CDCl}_{3}\right) \delta$ 1.00-1.47 $\left(10 \mathrm{H}, \mathrm{m}, \mathrm{NOCH}_{2} \mathrm{CH}_{3}\right.$, cyclopropyl-4H and $\left.-\mathrm{CH}_{3}\right)$, 3.27-3.52 $(5 \mathrm{H}, \mathrm{m}$, piperazinyl-5H $), 3.73-3.75(4 \mathrm{H}, \mathrm{m}$, piperazinyl-1 $\mathrm{H}$ and $\left.-\mathrm{OCH}_{3}\right), 4.03-4.05(1 \mathrm{H}, \mathrm{m}$, cyclopropyl$1 \mathrm{H}), 4.45-4.56\left(3 \mathrm{H}, \mathrm{m}, \mathrm{NOCH}_{2} \mathrm{CH}_{3}\right.$ and piperazinyl-1H), 5.24 $\left(2 \mathrm{H}, \mathrm{d}, J=12.0 \mathrm{~Hz},-\mathrm{CH}_{2}\right.$ - linker $), 5.30-5.36\left(2 \mathrm{H}, \mathrm{m},-\mathrm{CH}_{2}-\right.$ linker), 6.99-7.14 (2H, m, Ar-H), 7.19-7.88 (3H, m, Ar-H), $8.85(1 \mathrm{H}, \mathrm{s}, \mathrm{Ar}-\mathrm{H}), 14.69$ (1H, brs, COOH). HRMS-ESI: m/z Calcd. for $\mathrm{C}_{34} \mathrm{H}_{35} \mathrm{~F}_{2} \mathrm{~N}_{8} \mathrm{O}_{7} \quad[\mathrm{M}+\mathrm{H}]^{+}:$705.25913; Found: 705.25686 .

\subsection{1-cyclopropyl-7-(4-(2-(4-((3-(ethoxyimino)-5-} methyl-2-oxoindolin-1-yl)methyl)-1H-1,2,3-triazol1-yl)acetyl)-3-methylpiperazin-1-yl)-6-fluoro-8methoxy-4-oxo-1,4-dihydroquinoline-3-carboxylic acid (8n)

Yellow solid, yield: $69 \%$. ${ }^{1} \mathrm{H}$ NMR $\left(400 \mathrm{MHz}, \mathrm{CDCl}_{3}\right) \delta$ 1.28-1.50 (10H, m, $\mathrm{NOCH}_{2} \mathrm{CH}_{3}$, cyclopropyl-4H and $\left.-\mathrm{CH}_{3}\right)$, $2.27\left(3 \mathrm{H}, \mathrm{s},-\mathrm{CH}_{3}\right), 3.28-3.55(5 \mathrm{H}, \mathrm{m}$, piperazinyl- $5 \mathrm{H}), 3.73-$ $3.75\left(4 \mathrm{H}, \mathrm{m}\right.$, piperazinyl-1H and $\left.-\mathrm{OCH}_{3}\right), 4.01-4.03(1 \mathrm{H}, \mathrm{m}$, cyclopropyl-1H), $\quad 4.50-4.60 \quad\left(3 \mathrm{H}, \quad \mathrm{m}, \quad \mathrm{NOCH}_{2} \mathrm{CH}_{3}\right.$ and piperazinyl-1H), $5.08\left(2 \mathrm{H}, \mathrm{s},-\mathrm{CH}_{2}\right.$ - linker), 5.17-5.32 $(2 \mathrm{H}, \mathrm{m}$, $-\mathrm{CH}_{2}$ - linker $), 7.06(1 \mathrm{H}, \mathrm{d}, J=8.0 \mathrm{~Hz}, \mathrm{Ar}-\mathrm{H}), 7.20(1 \mathrm{H}, \mathrm{d}$, $J=8.0 \mathrm{~Hz}, \mathrm{Ar}-\mathrm{H}), 7.79$ (2H, s, Ar-H), $7.94(1 \mathrm{H}, \mathrm{d}, J=12.0$ $\mathrm{Hz}, \mathrm{Ar}-\mathrm{H}), 8.86(1 \mathrm{H}, \mathrm{s}, \mathrm{Ar}-\mathrm{H})$. HRMS-ESI: m/z Calcd. for $\mathrm{C}_{35} \mathrm{H}_{38} \mathrm{FN}_{8} \mathrm{O}_{7}[\mathrm{M}+\mathrm{H}]^{+}$: 701.28420 ; Found: 701.28039 .

\section{MIC determination}

The antibacterial activity of all hybrids against representative Gram-positive and Gram-negative strains were tested by means of standard two-fold serial dilution method using agar media. ${ }^{25}$ Petri dishes were incubated with $10^{4}$ colony-forming units (cfu) and incubated at $35^{\circ} \mathrm{C}$ for $18-24 \mathrm{~h}$.

\section{Cytotoxicity}

The cytotoxicity $\left(\mathrm{CC}_{50}\right)$ of the methylene and acetyl tethered gatifloxacin-1,2,3-triazole-isatin hybrids 8a-n, together with the parent gatifloxacin were examined by the 3(4,5-dimethylthiazol-2-yl)-2,5-diphenyltetrazolium bromide (MTT) assay in a mammalian VERO cells. ${ }^{26}$ The $\mathrm{CC}_{50}$ values were calculated by Bliss analyses.

\section{CONCLUSIONS}

In conclusion, fourteen methylene and acetyl tethered gatifloxacin-1,2,3-triazole-isatin hybrids 8a$\mathbf{n}$ were designed, synthesized and examined for their in vitro antibacterial activity against a panel of Grampositive and Gram-negative pathogens as well as cytotoxicity towards VERO cells. The synthesized hybrids showed promising in vitro activity against both drug-sensitive and drug-resistant organisms, and acceptable cytotoxicity towards VERO. Among them, hybrids $\mathbf{8 b}$ and $\mathbf{8 g}$ with highest activity against Gram-positive and Gram-negative pathogens respectively, also demonstrated acceptable cytotoxicity towards VERO cells. 
Acknowledgements. This study was supported by research grants from Key Research and Development Program of Liaoning Province $(2019 \mathrm{JH} 8 / 10300063)$ and Foundation of Liaoning Educational Department (2019-64).

\section{REFERENCES}

1. F. Gao, P. Wang, H. Yang, Q. Miao, L. Ma and G. M. Lu, Escherichia coli. Eur. J. Med. Chem., 2018, 157, 1223-1248.

2. Y. Q. Hu, S. Zhang, Z. Xu, Z. S. Lv, M. L. Liu and L. S. Feng, Eur. J. Med. Chem., 2017, 141, 335-345.

3. S. Zhang, Z. Xu, C. Gao, Q. C. Ren, C. Le, Z. S. Lv and L. S. Feng, Eur. J. Med. Chem., 2017, 138, 501-513.

4. Z. Xu, S. J. Zhao, Z. S. Lv, F. Gao, Y. L. Wang, F. Zhang, L. Y. Bai and J. L. Deng, Eur. J. Med. Chem., 2019, 162, 396-406.

5. G. F. Zhang, S. Zhang, B. F. Pan, X. F. Liu and L. S. Feng, Eur. J. Med. Chem., 2018, 143, 710-723.

6. C. Gao, Y. L. Fan, F. Zhao, Q. C. Ren, X. Wu, L. Chang and F. Gao, Eur. J. Med. Chem., 2018, 157, 1081-1095.

7. Z. Xu, X. F. Song, Y. Q. Hu, M. Qiang and Z. S. Lv, Eur. J. Med. Chem., 2017, 138, 66-71.

8. Z. Xu, S. Zhang, X. F. Song, M. Qiang and Z. S. Lv, Bioorg. Med. Chem. Lett., 2017, 27, 3643-3646.

9. D. Sriram, A. Aubry, P. Yogeeswari and L. M. Fisher, Bioorg. Med. Chem. Lett., 2006, 16, 2982-2985.

10. Z. Xu, S. Zhang, C. Gao, F. Zhao, Z. S. Lv and L. S. Feng, Chin. Chem. Lett., 2017, 28, 159-167.

11. D. Jiang, G. Q. Wang, X. F. Liu, Z. B. Zhang, L. S. Feng and M. L. Liu, J. Heterocyclic. Chem., 2018, 55, 1263-1279.

12. L. S. Feng, M. L. Liu, B. Wang, Y. Chai and H. Y. Guo, Eur. J. Med. Chem., 2010, 45, 3407-3412.
13. L. S. Feng, M. L. Liu, S. Zhang, Y. Chai, H. Y. Guo and C. L. Xiao, Eur. J. Med. Chem., 2011, 46, 341-348.

14. Z. Xu, Z. S. Lv, X. F. Song and M. Qiang, J. Heterocyclic Chem., 2018, 55, 97-102.

15. Z. Xu, X. F. Song, J. Fan and Z. S. Lv, J. Heterocyclic Chem., 2018, 55, 77-82.

16. Y. Q. Hu, Z. Xu, M. Qiang and Z. S. Lv, J. Heterocyclic Chem., 2018, 55, 187-191.

17. Z. Xu, S. J. Zhao, J. L. Deng, Q. Wang and Z. S. Lv, J. Heterocyclic Chem., 2019, 56, 319-324.

18. X. Yan, Z. Lv, J. Wen, S. Zhao and Z. Xu, Eur. J. Med. Chem., 2018, 143, 899-904.

19. C. Ji, P. A. Miller and M. J. Miller, ACS Med. Chem. Lett., 2015, 6, 707-710.

20. N. Purkayastha, S. Capone, A. K. Beck and D. Seebach, Chem. Biodivers., 2015, 12, 179-193.

21. R. Cormier, W. N. Burda, L. Harrington, J. Edlinger, K. M. Kodigepalli, J. Thomas, R. Kapolka, G. Roma, B. E. Anderson, E. Turos and L. N. Shaw, Bioorg. Med. Chem. Lett., 2012, 22, 6513-6520.

22. F. Gao, H. Yang, T. Y. Lu, Z. J. Chen, L. Ma, Z. Xu and G. M. Lu, Eur. J. Med. Chem., 2018, 159, 277-281.

23. Y. H. Zhang, R. Wang, T. Zhang, W. T. Yan, Y. H. Chen, Y. P. Zhang and M. Y. Zhou, Chin. Chem. Lett., 2019, 30, 653-655.

24. L. S. Feng, S. L. Hong, J. Rong, Q. C. You, P. Dai, R. B. Huang, Y. H. Tan, W. Y. Hong, C. Xie, J. Zhao and X. Chen, J. Am. Chem. Soc., 2013, 135, 9244-9247.

25. R. Wang, X. Yin, Y. Zhang and W. Yan, Eur. J. Med. Chem., 2018, 156, 580-586.

26. C. Gao, L. Chang. Z. Xu, X. F. Yan, C. Ding, F. Zhao, X. Wu and L. S. Feng, Eur. J. Med. Chem., 2019, 163, 404-412. 